\title{
MORE JORDAN TYPE INEQUALITIES
}

\author{
D. AHARONOV AND U. EliAS
}

Abstract. The function $\tan (\pi x / 2) /(\pi x / 2)$ is expanded into a Laurent series of $1-x^{2}$, where the coefficients are given explicitly as combinations of zeta function of even integers. This is used to achieve a sequence of upper and lower bounds which are very precise even at the poles at $x= \pm 1$.

Similar results are obtained for other trigonometric functions with poles.

Mathematics subject classification (2010): 26D05.

Keywords and phrases: Trigonometric inequalities, Jordan inequality, Laurent series, zeta function, Dirichlet functions.

\section{REFERENCES}

[1] M. Abramowitz And I. Stegun, eds., Handbook of Mathematical Functions with Formulas, Graphs, and Mathematical Tables, New York: Dover Publications, 1972.

[2] Á. BARICZ And S. Wu, Sharp Jordan-type inequalities for Bessel functions, Publ. Math. Debrecen, 74 (2009), 107-126.

[3] Á. BARICZ, Generalized Bessel Functions of the First Kind, Lecture Notes in Mathematics, Volume 1994, Springer Verlag, Berlin Heidelberg, 2010.

[4] M. BECKER AND E. STARK, On a hierarchy of quolynomial inequalities for $\tan x$, Univ. Beograd. Publ. Elektrotehn. Fak. Ser. Mat., Fiz 620 (1978), 133-138.

[5] C. P. Chen AND F. QI, Inequalities of some trigonometric functions, Univ. Beograd. Publ. Elektrotehn. Fak. Ser. Mat., 15 (2004), 71-78.

[6] F. QI, D. W. NiU AND B. N. GuO, Refinements, Generalizations and Applications of Jordan's inequality and related problems, J. of Inequalities and Applications, Volume 2009, Article ID 271923, 52 pages, doi:10.1155/2009/271293.

[7] R. L. Graham, D. E. Knuth and O. Patashnik, Concrete Mathematics, 2nd Edition, AddisonWesley, Reading, Massachusetts, 1994.

[8] J. L. LI, An identity related to Jordan's inequality, Int. J. Math. Math. Sci., vol. 2006, Article ID 76782, 6 pages, 2006. doi:10.1155/IJMMS/2006/76782.

[9] L. B. W. Jolley, Summation of series, 2nd edition, Dover Publication, N.Y., 1961.

[10] L. ZHU, Sharpening Jordan's inequality and the Yang Le inequality, Applied Mathematics Letters, 19 (2006), 240-243.

[11] L. ZHU, Sharpening Jordan's inequality and the Yang Le inequality, II, Applied Mathematics Letters, 19 (2006), 990-994.

[12] L. ZHU, A general refinement of Jordan-type inequality, Computers and Mathematics with Applications, 55 (2008), 2498-2505.

[13] L. ZHU, General forms of Jordan an Yang-Le inequalities, Applied Mathematics Letters, 22 (2009), 236-241.

[14] J. Spanier and Keith B. Oldham, An atlas of functions, Hemisphere Publishing, Washington, 1987. 